\title{
H1N1 INDUCED ARDS: ECMO AS RESCUE THERAPY IN PATIENTS WITH FAILED MECHANICAL VENTILATION - A REVIEW
}

\author{
Ismail AH*, Marzida $\mathbf{M}^{*}$, Kumar NM^, Ong Gracie $\mathrm{SY}^{*}$ \\ "Division of Cardiothoracic Surgery, Southern Railway Headquarters Hospital, Chennai, India. \\ *Department of Anaesthesiology, Faculty of Medicine, University of Malaya, Kuala Lumpur, Malaysia \\ ${ }^{\wedge}$ Division of Cardiac Anesthesia, Southern Railway Headquarters Hospital, Chennai, India.
}

\begin{abstract}
:
Since the outbreak of the novel influenza H1N1 in Mexico in April 2009, more than half a million cases have been recorded with close to 6000 deaths. In contrast to seasonal flu, this virus appears to have a predilection for the young, obese and pregnant. It's most important and almost fatal complication is Acute Respiratory Distress Syndrome (ARDS). ICUs around the world have scrambled to upgrade various treatment modalities including high frequency oscillation ventilation, inotropes, antivirals and antibiotics in an effort to reduce the mortality arising out of this complication. More importantly, this complication appears reversible if adequate and early therapy is instituted. In particular, rescue therapies that allow the lung to rest appear to have brought success in some clinical settings. This article describes the experiences of six centres that have used Extracorporeal Membrane Oxygenation (ECMO) as rescue therapy in patients having ARDS. ECMO has been instituted in many of these cases not only as a bridge to therapy but also to reduce further barotrauma in these patients. ECMO experiences regarding 2 patients at the University of Michigan, 7 in Canada, 68 patients at Leicester UK, 68 in Australia and New Zealand, 1 in Hong Kong and 2 in Singapore are described. (JUMMEC 2010; 13(2): 80-87)
\end{abstract}

KEYWORDS: Influenza, H1N1, ARDS, ECMO

\section{Introduction}

The novel influenza, H1N1 flu appears to be significantly different from seasonal influenza in that it kills much younger age group than ordinary flu according to the World Health Organization. As of 13 August, 2009, there have been more than 180,000 confirmed cases and nearly 1,800 deaths worldwide (1). The Centers for Disease Control (CDC) of the United States of America (US) announced in October 2009 that thus far 86 US children had died of H1N1 with most being in the 5 to 17-year-old age group and in severe cases, patients deteriorate in around three to five days following symptoms with deterioration being rapid, many progressing to respiratory failure within 24 hours (2). The World Health Organization declared the "first phase 6" global influenza pandemic of the century on 11 June, 2009 (3).
Unlike seasonal flu which generally is a disease of the upper respiratory tract, novel influenza H1N1 appears to have a greater predilection for the lower respiratory tract causing pneumonia. Although the exact role of obesity is poorly understood at present,
Correspondence:
Marzida Mansor
Department of Anaesthesiology
Faculty of Medicine
University of Malaya
50603 Kuala Lumpur, Malaysia
E-mail: marzida@ummc.edu.my 
obesity and especially morbid obesity have been present in a large portion of severe and fatal cases. Obesity has not been recognized as a risk factor in either past pandemics or seasonal influenza (2).

This review outlines the clinical features and experiences in seven centres or regions that utilised Extracorporeal Membrane Oxygenation (ECMO) as rescue therapy in patients who sustained acute respiratory distress syndrome (ARDS) as a complication of H1N1. ECMO experiences in two patients at the University of Michigan in the United States of America (US), seven in Canada, 68 patients at Leicester in the United Kingdom (UK), 68 in Australia and New Zealand, one in Hong Kong and two in Singapore are described.

\section{United States of America}

At the initial outbreak of novel influenza $A(H 1 N 1)$ in April 2009, the CDC reported the first two cases in the US (4). Sequelae of the viral infection included rapidly progressive lower respiratory tract disease resulting in respiratory failure, ARDS and prolonged intensive care unit (ICU) admission (3).

Between May and June 2009, the University of Michigan received 13 patients for evaluation, 10 of whom were confirmed to have novel influenza $A(\mathrm{H} 1 \mathrm{~N} 1)$ virus infection by testing of respiratory specimens with real-time reverse transcription-polymerase chain reaction ( $r R T-P C R)$. Of the 10, nine were obese (body mass index $[\mathrm{BMI}] \geq 30$ ), including seven who were extremely obese ( $\mathrm{BMI} \geq 40$ ); five had pulmonary emboli; and nine had multiorgan failure (5).

All ten patients were referred because of severe hypoxemia, ARDS and an inability to achieve adequate oxygenation with conventional ventilation modalities. The median age was 46 years (range: 21-53 years). There were three fatalities; the time from illness onset to death ranged from 17 to 30 days.

Nine patients were admitted for multiorgan failure and all nine manifested septic shock requiring vasopressor support. All 10 patients required tracheostomy. All patients received antibiotic therapy with oseltamivir and amantadine being administered beyond the standard 5-day course, including higherdose oseltamivir (up to $150 \mathrm{mg}$ orally twice a day).
All 10 patients required initial advanced mechanical ventilation (high-frequency oscillatory or bilevel ventilation with high mean airway pressures $32-55 \mathrm{~cm}$ $\mathrm{H} 20$ ). Two patients required veno-venous ECMO. Six required continuous renal replacement therapy (CRRT) for acute renal failure.

Of the 10 patients, one patient remained in ICU requiring ECMO, one remained on advanced mechanical ventilation, five were transferred back to the referring facility in stable condition, and three died. The two patients on ECMO were both male aged 43 and 51 years respectively. Both were on high-frequency oscillatory ventilation (HFOV) before being placed on ECMO. The 43-year-old had a BMI of 48.7 as compared to 39.7 for the 51-year-old. Both had multiorgan failure. However, the 43-year-old died while the 51-year-old survived to mechanical ventilation in ICU bringing the mortality for patients placed on ECMO for H1N1 induced ARDS in this series to $50 \%(5)$.

\section{Canada}

The Canadian Critical Care Trials Group (CCCTG) designed a multicenter observational study of critically ill patients infected with the 2009 influenza A (H1N1). Critically ill patients were defined as: (1) those admitted to a pediatric or adult ICU or those requiring mechanical ventilation (invasive or noninvasive), (2) those with a fraction of inspired oxygen $\left(\mathrm{FiO}_{2}\right)$ concentration greater than or equal to $60 \%$, or (3) those with the need for intravenous infusion of inotropic or vasopressor medication.

Anand Kumar et al gathered data in this prospective observational study of 168 critically ill patients with 2009 influenza A (H1N1) infection in 38 adult and pediatric ICUs in Canada between 16 April and 12 August, 2009. They identified 162 confirmed cases and six probable ones (6).

Critical illness occurred in 215 patients with confirmed $(n=162)$, probable $(n=6)$, or suspected $(n=47)$ influenza $A(H 1 N 1)$ infection. Among the 168 patients the mean age was 32.3 years; 113 were female (67.3\%) and 50 were children (29.8\%). Overall mortality among critically ill patients at 28 days was $14.3 \%$. A third of the patients were obese, and $67 \%$ were female, a gender imbalance that was unexplained. 
Fifty-one patients had major underlying conditions, with chronic lung disease, obesity, hypertension, and smoking the most common. Evidence of bacterial pneumonia was seen in $24 \%$.

Neuraminidase inhibitors were administered to 152 patients (90.5\%). All patients were severely hypoxemic (mean ratio $\mathrm{PaO}_{2}: \mathrm{FiO}_{2}$ of 147 ) at ICU admission. Mechanical ventilation was received by 136 patients (81.0\%). The median duration of ventilation was 12 days and ICU stay was 12 days.

The mean daily positive end-expiratory pressure (PEEP) was greater than $10 \mathrm{~cm} \mathrm{H_{2 }} \mathrm{O}$ for the first two weeks of mechanical ventilation. Over the first two weeks of critical illness, tidal volumes ranged from 8 to $9.1 \mathrm{ml} / \mathrm{kg}$ of body weight; and carbon dioxide elimination was not substantially impaired. Barotrauma occurred in 14 patients (8.3\%). Therapies for oxygenation failure included neuromuscular blockade (47 patients; $28.0 \%$ ), inhaled nitric oxide (23 patients; $13.7 \%)$, high-frequency oscillatory ventilation (20 patients; 11.9\%), extracorporeal membrane oxygenation (seven patients; $4.2 \%$ ), and prone positioning ventilation (five patients; $3.0 \%$ ).

Six patients were placed on ECLS in four ICUs due to profound hypoxemia unresponsive to less invasive therapeutic interventions. Four patients were supported with veno-venous pump-driven ECMO and two were supported with pumpless lung assist (Novalung iLA). Average duration of support was 15 days. Four patients survived (66.6\%)-one patient supported with iLA and three patients supported with ECMO.

The authors concluded that severe illness arises in a young, previously healthy population with a high probability of survival given the availability of appropriate resources and this has important societal implications. If, as expected, the prevalence of 2009 influenza $A(\mathrm{H} 1 \mathrm{~N} 1)$ infection increases with the upcoming flu season, there will be an acute increase in demand for ICU care, including the need for rescue therapies that are not currently widely available (7-9). Clinicians and policy makers will need to examine feasible methods to optimally expand and deploy ICU resources including possibly ECMO to meet this need.

\section{United Kingdom}

Giles Peek and associates at Leicester aimed to delineate the safety, clinical efficacy, and cost-effectiveness of ECMO compared with conventional ventilation support in severe acute respiratory failure in adults who have a high mortality despite improvements in ventilation techniques and other treatments (eg, steroids, prone positioning, bronchoscopy, and inhaled nitric oxide). (10).

They randomly assigned 180 adults to conventional management or referral to consideration for treatment by ECMO. Eligible patients were aged 18-65 years and had severe (Murray score $>3.0$ or $\mathrm{pH}<7.20$ ) but potentially reversible respiratory failure. Exclusion criteria were: high pressure $\left(>30 \mathrm{~cm} \mathrm{H} \mathrm{H}_{2} \mathrm{O}\right.$ of peak inspiratory pressure) or high $\mathrm{FiO}_{2}(>0.8)$ ventilation for more than 7 days; intracranial bleeding; any other contraindication to limited heparinization.

766 patients were screened; 180 were enrolled and randomly allocated to consideration for treatment by ECMO ( $\mathrm{n}=90$ patients) or to receive conventional management $(\mathrm{n}=90) .68$ (75\%) patients actually received ECMO; 63\% (57/90) of patients allocated for treatment by ECMO survived to 6 months without disability compared with $47 \%(41 / 87)$ of those allocated to conventional management

They recommend transferring of adult patients with severe but potentially reversible respiratory failure, whose Murray score exceeds 3.0 or who have a pH of less than 7.20 on optimum conventional management, to a centre with an ECMO-based management protocol to significantly improve survival without severe disability.

\section{Australia and New Zealand}

In Australia, Davies et al reported that most patients who underwent extracorporeal membrane oxygenation (ECMO) for respiratory failure survived their struggle with pandemic $\mathrm{H} 1 \mathrm{~N} 1$ flu. Of the 68 patients treated with ECMO during the winter, 54 were still alive as of 7 September, 2009. They suggest that their results should aid healthcare planning and clinical management for these complex patients during the ongoing pandemic (11). 
They examined data from all 68 patients treated with ECMO in Australia and New Zealand at the 15 ICUs providing the service. The study found that 68 patients with severe influenza-associated ARDS were treated with ECMO. An additional 133 patients with influenza $A$ received mechanical ventilation, but not ECMO, in the same ICUs. Of those, 53 had laboratory-confirmed pandemic H1N1, while eight had confirmed infection with nonsubtyped influenza $A$. The other seven were regarded as having suspected infection with the pandemic strain.

The authors estimated the incidence of ECMO use for confirmed and suspected $\mathrm{H} 1 \mathrm{~N} 1$ to be 2.6 per one million people. Assuming a similar incidence of ECMO use for the current flu season in the Northern Hemisphere, the US and Europe might expect to provide treatment for 800 and 1,300 patients respectively. The median age of the patients in their study was 34.4, and half were male. Three children younger than 15 received ECMO. None of the patients was older than 65 .

Again, the most common comorbidities were obesity in $50 \%$, asthma in $28 \%$, and diabetes in $15 \%$. Nearly one in 10 was pregnant. More than a quarter (28\%) were coinfected with a bacterium, most commonly Streptococcus pneumoniae and Staphylococcus aureus.

Prior to institution of ECMO, about two-thirds (68\%) of patients received vasoactive drugs and $24 \%$ received renal replacement therapy. Almost all of the patients (94\%) received oseltamivir (Tamiflu) for a median duration of eight days. The median duration of ECMO was 10 days. Most patients (81\%) received rescue therapies for acute respiratory distress syndrome, including recruitment maneuvers (67\%), inhaled nitric oxide (32\%), epoprostenol (22\%), prone positioning (20\%), and high-frequency oscillatory ventilation $(5 \%)$.

Hemorrhagic complications were common, occurring in $54 \%$ of ECMO patients. The most common sources were ECMO cannulation sites $(22 \%)$, the gastrointestinal tract $(10 \%)$, and the respiratory tract (10\%). As of 7 September, 2009 , some $21 \%$ of patients who received ECMO had died. Of the survivors, 32 had been discharged from the hospital, 16 were still hospitalized but no longer in the ICU, and six remained in the ICU.

Clinicians in Australia were "forewarned" of what to expect before swine flu arrived in Australia, by the experiences of doctors in Mexico and the US. When the outbreak of swine flu was announced in Mexico, many young people were presenting with severe pneumonia. They also reported a very high fatality rate per case of infection, but stressed that the result from insufficient planning and inadequate preparation despite the virus' tendency to cause potentially reversible ARDS, would be tragic. They suggest that ECMO should be considered when an adult with respiratory failure has a $50 \%$ chance of dying and is indicated if there is an $80 \%$ chance of dying using conventional algorithms that take into account blood gases, ventilator pressure, the extent of shock, and other factors.

\section{Hong Kong}

In Hong Kong, Liong and associates reported one case of ECMO done on a 37-year-old Filipino woman. Hong Kong had more than 10,000 cases of the virus confirmed by 27 August, 2009. 44 were in serious condition and four had died, 17 had recovered and discharged (12, 13).

The Filipino woman had presented with fever and failed to respond to treatment by her general practitioner. She complained of influenza like symptoms and was subsequently hospitalized 10 days after the onset of symptoms where she was found to have severe pneumonia associated with severe hypoxemia. Her initial chest X-ray (CXR) showed bilateral lung airspace consolidation. She was admitted directly to ICU and placed in respiratory isolation.

A nasopharyngeal aspirate (NPA) was negative for influenza $A$ and $B$ rapid antigen, as was the real-time reverse-transcriptase polymerase chain reaction (RT$P C R)$ for novel influenza $A(H 1 N 1)$ on her throat and nasal swab. She was commenced on imipenem/cilastatin and azithromycin, but her condition deteriorated rapidly and required intubation and mechanical ventilatory support the day after admission.

Influenza $\mathrm{A}(\mathrm{H} 1 \mathrm{~N} 1)$ infection was confirmed when her initial aspirates tested positive after a second round of testing. Oseltamivir $75 \mathrm{mg}$ twice daily was 
started on day 3 of admission. The dose of oseltamivir was increased to $150 \mathrm{mg}$ twice daily on day 4 of admission and was continued for 18 days. Zanamivir was also given as the patient was critically ill and did not respond well to oseltamivir as there was concern about oseltamivir resistance.

She was given nebulised zanamivir $15 \mathrm{mg}$ diluted in $2 \mathrm{ml}$ normal saline for 4 doses over 3 days. It was discontinued following severe desaturation related to loss of PEEP. Despite aggressive treatment, she had refractory hypoxaemia with a $\mathrm{PaO}_{2}$ of $52 \mathrm{~mm}$ $\mathrm{Hg}$ while receiving ventilatory support on an $\mathrm{FiO}_{2}$ of 1.0 , inspiratory pressure of $35 \mathrm{~cm} \mathrm{H}_{2} \mathrm{O}$ and PEEP of $20 \mathrm{~cm} \mathrm{H} \mathrm{H}_{2} \mathrm{O}$. Veno-venous extracorporeal membrane oxygenation (VV-ECMO) was instituted on day 7 of her admission. On commencement of VV-ECMO, the patient had no other vital organ failure and her oxygenation improved initially.

However, she could not be weaned to a lower level of ventilatory support to prevent barotrauma. The patient had clinical evidence of ventilator-associated pneumonia on day 12. She developed septic shock requiring inotropic support and acute renal failure requiring intermittent renal replacement therapy. She subsequently developed haemolysis, which precluded the use of VV-ECMO which was stopped. She succumbed eventually the same day. A lung autopsy specimen revealed ARDS.

Although the authors maintain that VV-ECMO is an effective way to support patients with refractory hypoxaemia who fail to respond to conventional ventilatory strategies as reported by Gattinoni, the CESAR trial and Nehra, they indicated that they faced serious diagnostic problems in the beginning with this patient as all tests regarding the novel influenza returned as negative. They suggest clinicians maintain a high level of suspicion for ARDS brought on by H1N1 $(14,15,16)$.

They concluded that ECMO is not a risk-free procedure as it can cause severe haemolysis, hemorrhage, and haemodynamic instability. It is also labor-intensive and technically demanding and is unrealistic and too costly to provide this procedure to every critically ill patient with influenza $\mathrm{A}(\mathrm{H} 1 \mathrm{~N} 1)$ in the midst of a pandemic when the demand for critical care services have already been stretched to the limit.

\section{Singapore}

The Singapore General Hospital reported two cases that underwent ECMO as a result of patients coming down with H1N1 induced ARDS. The first was a 36-year-old security guard who was placed on ECMO when he failed to respond to conventional mechanical ventilation therapy. He was subsequently decannulated a week later to ventilation therapy before recovering. He was eventually discharged 17 days after admission ,but with residual decrease in effort tolerance. The second patient was a 20-year-old lady who was being treated at the National University Hospital. Clinicians have noted that like elsewhere, $\mathrm{H} 1 \mathrm{~N} 1$ is peculiar in that it is young people who appear to progress rapidly to respiratory distress (17).

\section{Discussion}

Since 1972 when Hill et al (18) reported the survival of a 24-year-old polytraumatized patient with ARDS treated with ECMO and following four years later with Bartlett et al (19) reporting the survival of the first newborn treated with ECMO, many research groups have tried utilizing ECMO for both cardiac and respiratory failure. However interest in the technique as a treatment modality faded when the $\mathrm{NIH}$ sponsored randomized multicenter trial in 1974 to test venoarterial ECMO versus conventional therapy in adult ARDS patients (20) revealed that mortality rates in the ECMO therapy group were as high as $90 \%$ and not significantly different from those in the conventionally treated group.

The idea of supporting impaired lung function with extracorporeal gas exchange in adults, however, was subsequently pursued by Kolobow et al (21). The rationale of their advanced technique was to prevent further damage to the diseased lungs by reducing their motion (pulmonary rest) with application of only a few ventilator breaths with low VT and low peak inspiratory pressures. This lung protective mechanical ventilation strategy became known as low-frequency positivepressure ventilation (LFPPV) (14).

With this method, oxygen uptake and carbon dioxide removal were dissociated. Oxygenation was primarily accomplished through the nearly motionless natural lung via apneic oxygenation, and carbon dioxide was cleared through the artificial lung [extracorporeal carbon dioxide removal $\left.\left(\mathrm{ECCO}_{2}-\mathrm{R}\right)\right]$. The so-called 
LFPPV-ECCO 2 -R technique was performed at low extracorporeal blood flows (20-30\% of cardiac output), so that a venovenous bypass technique instead of an arteriovenous one sufficed, which turned out to be less detrimental to blood cells, coagulation and internal organs.

Using LFPPV-ECCO $2-\mathrm{R}$, Gattinoni et al reported survival rates of up to $49 \%$ (14). In the following years several centres corroborated promising survival rates of around $50 \%$ and higher. The utilization of ECMO has again gained prominence in lieu of the severe and rapid ARDS that affects patients with novel influenza H1N1.

Although there are many conservative measures that include a no prone position, aggressive diuresis/ ultrafiltration, HFOV, shunt reduction manoeuvres (high mean airway pressures, inhaled nitric oxide and maybe even the use of prostacyclin), ECMO does have a role if all of these manoeuvres fail in the face of worsening barotrauma especially in young patients with a potentially reversible disease such as the novel H1N1. The Australian and CESAR studies suggest that ECMO is a modality that can indeed save lives as a rescue therapy when conventional methods fail $(10,11)$.

However, ECMO is not without its problems. It can be technically difficult in obese patients. And as indicated by Liong and associates, haemolysis could be a problem especially in a patient with multiorgan failure. This could potentially tax a blood bank if platelets and other coagulation factors need to be constantly corrected to maintain appropriate levels of these blood components. Furthermore, bleeding from cannulation sites can be a problem. It further requires a highly dedicated and committed team. And there is a learning curve that is associated with providing this service which will require training of personnel $(13,22)$.

The Australian and CESAR trials however, have indicated that ECMO should be considered for most patients who cannot receive lung-protective ventilation and should be implemented early, possibly before 7 days. Even if only a tiny portion of $\mathrm{H} 1 \mathrm{~N} 1$ patients get sick enough to need ECMO, the anticipated demand in the wake of pandemic influenza $A(\mathrm{H} 1 \mathrm{~N} 1)$ threatens to overwhelm the facilities currently equipped with this technology. ECMO should be considered when an adult with respiratory failure has a $50 \%$ chance of dying and is indicated if there is an $80 \%$ chance of dying using conventional algorithms that take into account blood gases, ventilator pressure, the extent of shock, and other factors. Survival rates for patients with severe respiratory failure can approach $60 \%-70 \%$ if ECMO is used early on $(10,11,22)$.

Cost is another issue concerning this technology. The current cost of ECMO is approximately US\$2,000/day, but that is expected to drop as the newer technology gets more widely adopted. In fact, advances in technology in the past few years have made ECMO simpler and less expensive than in the past, and data from the CESAR trial showed a survival benefit using ECMO rather than conventional ventilation support for severe adult respiratory failure $(10,11,22)$.

With the winter almost here in the Northern Hemisphere, there could be resurgence in respiratory failure secondary to viral necrotizing pneumonia related to $\mathrm{H} 1 \mathrm{~N} 1$ influenza that will increase demand for ECMO. The Cesar trial has already established the use of ECMO during the first wave of the pandemic with good effect and it has further proven to be an invaluable weapon in the fight against the winter resurgence of the infection, as has already been seen during the Australasian winter $(10,11,22)$.

In Malaysia, Sam et al have however reported that of the $\mathrm{H} 1 \mathrm{~N} 1$ dead, 50\% were previously healthy with the rest having underlying conditions (23). Malaysia has thus far recorded 7,066 cases and 77 deaths, with most deaths presumably being due to necrotizing ARDS (24). Data regarding the predilection among the sexes or obesity is unavailable. The Canadian, Leicester and Australian studies in particular are important lessons in the local context that anesthetists and critical care specialists could perhaps ensure that ICU facilities and especially rescue therapies such as high-frequency oscillatory ventilation, nitric oxide and even perhaps ECMO should be made available to treat patients with the novel influenza $\mathrm{H} 1 \mathrm{~N} 1(6,10,11)$.

\section{Conclusion}

H1N1 can produce a rapidly progressive respiratory failure that is refractory to conventional mechanical 
ventilation, often in young, healthy patients-a group who are not currently a priority group for H1N1 vaccination $(25,26)$. The rapid onset of refractory hypoxemia, together with multisystem organ failure and hypotension, suggests that clinical outcomes will depend on clinicians' ability to apply sophisticated mechanical ventilatory support and adjunct therapies. The most serious of its complications, rapid ARDS, appears potentially reversible if patients are triaged, categorized and treated early. The issue of trying to treat patients who have sustained barotrauma or those presenting late without a facility such as ECMO is a difficult one. Unlike the 1918 Spanish Flu (27-30), this pandemic's mortality appears to have been curtailed by the availability of antivirals, antibiotics, vasopressors, ventilators and better ICU care. ECMO could be another step to further reduce this mortality.

\section{References}

1. Pandemic (H1N1) update 62 (revised 21 August 2009). Laboratory-confirmed cases of pandemic (H1N1) 2009 as officially reported to WHO by States Parties to the IHR (2005) as of 13 August 2009. Available from http: //www.who.int/csr/ don/2009_08_21/en/index.html. Accessed 21 Aug 2009.

2. New flu can kill fast, researchers agree. Maggie Fox Reuters, Oct 16, 2009, Health and Science Editor.

3. Perez-Padilla $R$, de la Rosa-Zamboni $D$, Ponce de Leon $S$, et al. Pneumonia and respiratory failure from swine-origin influenza $\mathrm{A}(\mathrm{H} 1 \mathrm{~N} 1)$ in Mexico. $N$ Engl J Med 2009.

4. CDC. Swine influenza $A(H 1 N 1)$ infection in two children-Southern California, March-April 2009. MMWR 2009; 58: 400-2.

5. LM Napolitano, PK Park, KC Sihler, et al. IntensiveCare Patients With Severe Novel Influenza A(H1N1) Virus Infection - Michigan, June 2009. July10, 2009. MMWR on MMWR website.

6. Kumar A, Zarychanski R, Pinto R, et al. Critically ill patients with 2009 influenza $A(H 1 N 1)$ infection in Canada. JAMA 2009 Oct 12; 302(17).

7. OSCAR Trial Website. High frequency oscillation in ARDS. Available from http://duncanyoung.net/ index.php. Accessed 20 July, 2009.
8. Meade MO, Cook DJ, Mehta S, et al. A multicenter pilot randomized trial of high frequency oscillation in acute respiratory distress syndrome. Am J Respir Crit Care Med 2009; 179: A1559.

9. Adhikari NK, Burns KE, Friedrich JO, et al. Effect of nitric oxide on oxygenation and mortality in acute lung injury: systematic review and meta-analysis. BMJ 2007; 334(7597): 779-782.

10. Giles J. Peek of Glenfield Hospital, Leicester, U.K., and his associates in the Conventional Ventilation or ECMO for Severe Adult Respiratory Failure (CESAR) trial. Lancet 2009 Sept. 16 [doi: 10.1016/ S0140-6736(09)61069-2]).

11. Davies A, Beca J, Bellomo R, et al Extracorporeal membrane oxygenation for 2009 influenza $\mathrm{A}(\mathrm{H} 1 \mathrm{~N} 1)$ acute respiratory distress syndrome. JAMA 2009 Oct 12; 302(17).

12. Update on human swine influenza. HKSAR Centre for Health Protection website. Available from http://www.chp.gov.hk/view_content.

13. Liong T, Lee KL, Poon YS, et al.The first novel influenza $A(H 1 N 1)$ fatality despite antiviral treatment and extracorporeal membrane oxygenation in Hong Kong. Hong Kong Med J 2009; 15: 381-384.

14. Gattinoni L, Pesenti A, Mascheroni D, et al. Low frequency positive-pressure ventilation with extracorporeal $\mathrm{CO}_{2}$ removal in severe acute respiratory failure. JAMA 1986; 256: 881-886.

15. Hitt E. CESAR Trial: Extracorporeal Membrane Oxygenation Improves Survival in Patients with Severe Respiratory Failure. Proceedings of the Society of Critical Care Medicine 37th Critical Care Congress: late-breaking session. 2008 Feb 2-6. Honolulu, Hawaii.

16. Nehra D, Goldstein AM, Doody DP, et al. Extracorporeal membrane oxygenation for non-neonatal acute respiratory failure: the Massachusetts General Hospital experience from 1990 to 2008. Arch Surg 2009; 144: 427-32.

17. He turned blue \& needed machine to breathe for him. Oct 11, 2009 The New Paper.

18. Hill JD, O'Brien TG, Murray JJ, et al. Prolonged extracorporeal oxygenation for acute post- 
traumatic respiratory failure (shock-lung syndrome). N Engl J Med 1972; 286: 629-634.

19. Bartlett $\mathrm{RH}$, Gazzaniga $A B$, Jefferies $M R$, et al. Extracorporeal membrane oxygenation (ECMO) cardiopulmonary support in infancy. Trans Am Soc Artif Intern Organs 1976; 22: 80-93.

20. Zapol WM, Snider MT, Hill JD, et al. Extracorporeal membrane oxygenation in severe acute respiratory failure. A randomized prospective study. JAMA 1979; 242: 2193-2196.

21. Kolobow T, Gattinoni L, Tomlinson T, et al. Control of artificial breathing using an extracorporeal membrane lung. Anesthesiology 1977; 46: 138-141.

22. Flu Pandemic Pushing Demand for ECMO, Sherry Boschert Elsevier Global Medical News, October 8, 2009.

23. Sam IC, Abu Bakar S. Pandemic Influenza $A(H 1 N 1)$ 2009 in Malaysia - The next phase. Med J Malaysia Vol 64 No 2 June 2009.

24. Swine Flu Count - Worldwide statistics of the H1N1 Influenza A. Available from http://www.flucount. org/. Accessed 2009
25. Douglas BW, Angus DC. Preparing for the Sickest Patients with 2009 Influenza A(H1N1) JAMA 2009; 302(17) : (doi:10.1001/jama.2009.1539).

26. US Centers for Disease Control and Prevention. $2009 \mathrm{H} 1 \mathrm{~N} 1$ vaccination recommendations. Available from http://www.cdc.gov/h1n1flu/ vaccination/acip.htm. Accessed Aug 2009.

27. Oxford JS. Influenza A pandemics of the 20th century with special reference to 1918: virology, pathology and epidemiology. Rev Med Virol 2000; 10(2): 119-133.

28. Morens DM, Fauci AS. The 1918 influenza pandemic: insights for the 21st century. J Infect Dis 2007; 195(7): 1018-1028.

29. Cox NJ, Subbarao K. Global epidemiology of influenza: past and present. Annu Rev Med 2000; 51: 407-421.

30. Starr I. Influenza in 1918: recollections of the epidemic in Philadelphia. Ann Intern Med 2006; 145(2): 138-140. 